\title{
Strategic Tabletop Negotiations
}

\author{
Tokuo Yamaguchi ${ }^{1}$, Sriram Subramanian ${ }^{2}$, \\ Yoshifumi Kitamura ${ }^{1}$, and Fumio Kishino \\ ${ }^{1}$ Human Interface Engineering Laboratory, Osaka University, \\ Suita, Osaka 565-0871, Japan \\ ${ }^{2}$ Department of Computer Science, University of Saskatchewan, \\ 110 Science Place, Saskatoon, Saskatchewan, Canada S7N 5C9 \\ \{yamaguchi.tokuo, kitamura, kishino\}@ist.osaka-u.ac.jp, \\ sriramecs.usask.ca
}

\begin{abstract}
Strategic negotiations in digital tabletop displays have not been well understood. There is little reported in the literature on how users strategize when group priorities and individual priorities conflict and need to be balanced for a successful collaboration. We conducted an observational study on three digital tabletop systems and a real-world setup to investigate similarities and differences in real-world and digital tabletop strategic collaborations. Our results show that in the real world, strategic negotiation involves three phases: identifying the right timing, using epistemic actions to consider a task plan and evaluating the value of the negotiation. We repeated the real-world experiments with different digital tabletops and found several differences in the way users initiate and perform strategic negotiations.
\end{abstract}

Keywords: Face-to-Face Collaboration, Digital Tabletops, Strategic Negotiations, Collaborative Tables, Single Display Groupware.

\section{Introduction}

When working in groups, we often conduct face-to-face meetings to accelerate the exchange of ideas or opinions, or to complete a cooperative task. Many studies and systems have been proposed to facilitate face-to-face collaboration during group meetings or discussions [4, 6, 11, 16-18].

Collaboration may be sought personally or imposed managerially. A variety of collaborative situations arises in everyday situations and has been analyzed by many researchers (e.g. [1, 21]). Some types of collaborative tasks, especially in business situations, fundamentally include both competitive and collaborative aspects. For example, at a meeting where sports team managers gather to trade baseball players, each participant attempts to increase individual benefit while the final goal of the meeting is prosperity of the sporting world. Similar examples can be found in a meeting on reshuffling of personnel in a company or an organization where managers or directors of some sections participate. Other examples can be found in various trading floor-like and auction scenarios.

In a collaborative task that includes both competitive and cooperative aspects, one of the fundamental actions of participants is to achieve their desired results through 
negotiation with other participants. A participant has to observe the transition of the task and find the ripest timing and best partner with whom to negotiate. Through the negotiation with a particular partner, each participant attempts to increase his individual benefit and this leads to an increase in the group's benefit. This process can be called "strategic negotiation" and is complex because conflicts between the personal and group priorities frequently arise. While it has been discussed in business situations (e.g. [3]), there is no investigation of this aspect with respect to designing tabletop systems.

Strategic negotiations in face-to-face collaboration require users to support transitions between personal and group work, and when we negotiate with others while sharing information, we often have to be careful about which parts of the information can and cannot be shared [2]. It offers them the opportunity to consult hidden information to make an informed decision or to present information at the most appropriate time to maximize its impact and increase its value to the presenter. However, digital tabletops today are either clumsy or not capable of effectively handling strategic negotiations. A special framework is often required to allow multiple users to deal with private as well as public information.

To design better digital tabletops, we need to have an understanding of the lowlevel dynamics of user actions that accompany strategic negotiations. We conducted an observational study to investigate strategic negotiations in various digital tabletop settings. Our results show that in the real world, strategic negotiation involves three phases: identifying the right timing, using epistemic actions to draw attention and evaluating the value of the negotiation. We repeated the real-world experiments in different digital tabletops and found several differences in the way users initiate and perform strategic negotiations. We identify many implications for the design of digital tabletops that arise from our findings.

\section{Related Work}

Recently, there has been a proliferation of systems and techniques that support digital tabletop interactions. Here we review the literature in two related areas - we present previous efforts in prototyping novel tabletop systems and investigations into the dynamics of face-to-face collaboration and various tabletop designs for managing user privacy in public information spaces.

\subsection{Tabletop Systems}

From time immemorial, tables have been used to discuss and make important decisions by a group of co-located people. The example of King Arthur's fabled Round Table still persists in the popular imagination. Today, we often have discussions while standing or sitting around a table to accelerate the exchange of ideas with multiple persons. Focusing on this type of interaction, there is much literature devoted to interactive tabletop displays to support face-to-face cooperative works. For example, InteracTable allows a group to annotate digital content on a computationally-enhanced table [17], and DiamondTouch is a touch-sensitive tabletop display for multiple users [4]. ConnecTables allows users of combined mobile desks 
to create a larger horizontal workspace and share and exchange documents, and a rapid sub-grouping in an office environment can be elegantly achieved [18]. An approach to tangible interface that uses phicons and phandles on the tabletop can be found in metaDESK [22] and Sensetable [9]. Augmented Surfaces [11] is an example of a shared continuous workspace that combines walls, tabletops and laptops. Other tabletop displays are surveyed in [15].

Many researchers have investigated tabletop collaboration and proposed some characteristics as foundations for the design of interaction techniques. Pinelle et al. [10] propose the mechanics of collaboration as a set of low-level actions and interactions that must be supported if team members are to accomplish a task in a collaborative fashion. Basic actions include communication, coordination, planning, monitoring and protection. Kruger et al. [7] studied the role of spatial orientation on communication and collaboration, through observational studies of collaborative activity at a traditional table. They found that orientation is important in establishing personal and group spaces and in signaling ownership of objects. Ryall et al. [12] explored the effect of table size and number of collaborators on collaboration. They found that even larger groups were successfully able to manage work at a small table. In order to avoid interference, collaborators usually separated the workspaces based on their seating positions and the task semantics [20]. Scott et al. [13, 14] took a closer look at how territoriality affects collaboration in tabletop workspaces. They found that three types of territories were common - personal, group, and storage territories - and that these spatial divisions helped coordinate people's activities in shared tasks. Recently, Tang et al. [19] investigated various forms of collaborative coupling, the manner in which collaborators are involved and occupied with each other. They identified six distinct styles of coupling based on whether users work in the same area of the shared workspace or if they perform similar tasks.

Most of the above research focused on designing systems for face-to-face cooperation and understanding users' pragmatic actions in cooperative settings. Most of the collaboration characteristics describe physical actions that allow users to cooperate better. It is not clear if these findings transfer directly to strategic collaborations. There is no reported investigation on the dynamics or characteristics of managing the various spaces during a strategic negotiation.

\subsection{Privacy in Public Information Spaces}

The space between users can be broadly divided into private, personal, shared and public spaces. Private space can be defined by the area where the owner can see and manipulate data but others cannot see the data or observe the owner's detailed manipulations of the data; personal space is the area where the owner can see and manipulate data while at the same time other users can observe the owner's actions in that area (without being able to observe the details of the data); a public space is defined by the area that allows all users to see and manipulate all data in it; a shared space is a form of public space that is created for a specific subgroup of users.

Because all the pieces of information displayed on the aforementioned tabletops can be easily observed by all participants equally, these systems do not provide appropriate support for strategic negotiations. For the purpose of strategic negotiations, a participant should be allowed to maintain parts of the information in a 
private space that is not readily observable by other participants until the opportunity is ripe for sharing all or part of that private information. The next paragraph describes digital tabletop examples that protect private information in public spaces.

In Augmented Surfaces [11], the authors support a private space that is integrated with the public space through an interaction technique called hyperdragging. Users can control which space to put the information in by using an amplified dragging gesture to move data between the private and public spaces. In RoomPlanner, users can create a private space on the table in front of them using a hand gesture that physically occludes the information from the other user's view [23]. The Lumisight Table provides different images to different users around the tabletop and has private spaces for individual users but lacks a public space in which information can be shared by multiple users [8]. UbiTable allows two users to transfer digital media from their laptops (private space) to a tabletop display (public space) where it can be shared and annotated [16]. It also includes a personal space along the boundary between the private and public space where users can observe each other's actions. Storage Bins is a mobile storage mechanism that enables access to stored items anywhere on the tabletop [14]. The electronic pile of stored items may be useful for hiding information from the other users and relies on social protocols to prevent others from manipulating them. A tabletop display system that allows multiple users to interact with both private and public information on a shared display in a face-to-face setting was proposed in [6]. The system relies on head tracking and users' viewpoints to dynamically create private spaces that can overlap with other users' private spaces to create shared and public spaces.

While system designers have built several tables that support private and public spaces, investigations into tabletop collaboration suggest the need for dynamic personal and public spaces. It's not clear from the literature what the interplay is between private, public and personal spaces with regards to strategic negotiations.

\section{Study of Strategic Negotiations in Real World}

In order to better understand the characteristics and factors that influence strategic negotiations during face-to-face collaboration, we conducted an observational study in various collaborative settings at a real-world table. The results of this study were used to help focus our study of a digital table.

\subsection{Task and Method}

We designed three strategic tasks for groups of four to six people at a single table. The tasks were designed to leverage various types of digital tabletop settings. We were interested in three types of negotiations: public-space negotiations like on an auction floor, shared-space negotiations like on a trading floor, and shared-public negotiations like in a boardroom meeting.

Task 1: Here participants build a story based on a given theme on a large sheet by linking 10 images into a storyboard. Each participant was given 10 different images 
from which they had to select five to help build the story. After selecting their images, each participant had to convince the others to use as many of their five selected images as possible to create the story line. Since there were only 10 spots available for the storyboard, not all images from all participants made their way into the storyboard. Participants were instructed to build the storyboard with the intention of using as many of their images as possible. This task requires users to negotiate strategically in the public space where everyone could observe and interact with everyone else.

Task 2: The primary objective of this task was to examine strategic negotiations when users have to perform shared-public negotiations with several different partners at a table. The task is similar to the first one, but participants build two small stories and combine them into a big story by adding an image at the end. As with Task 1, after selecting their five images, participants are divided into two groups and asked to build two small stories. In this task, the story built by each group must have eight images, so each participant has to convince the others to use as many of their own five selected images as possible for the storyboard. Then, one image out of the remaining images has to be selected to serve as a link between the two stories. Participants try to convince each other that one of their own images best represents the two stories. This task requires the users to negotiate with partners in a dynamically created shared space followed by negotiations in the public space.

Task 3: The third task was a card game called "Pit" that uses 52 playing cards. Each participant starts with five cards and one card is placed face-up in the center of the table. Participants take turns exchanging cards to acquire a hand consisting of five cards of a similar suit (a flush in Poker). There are two ways to exchange cards participants can either swap one of their own cards for the card in the center of the table or can choose another participant with whom to negotiate and trade. In the second case, either of the participants can reject the negotiation before the transaction is completed. This game examines strategic negotiations when partners use private spaces in conjunction with shared spaces to engage with negotiating partners.

Participants and Method: Six groups participated in this study in a between participants design. For Tasks 1 and 2, each group consisted of four participants. Task 3 consisted of one group of four and one group of six participants. All 26 participants were university students aged 24-30 and included both females and males of varying ethnic origins. Prior to the task, each group received instructions on how to perform the task. Tasks 1 and 2 lasted 15 minutes each, whereas Task 3 lasted approximately 30 minutes. During each task, participants were comfortably seated around the table.

All sessions were videotaped and analyzed to compare and contrast the sharing of both the tabletop workspace and the objects on the table.

\subsection{Results and Discussion}

Figure 1 shows a snapshot of each task. Based on the analysis of the video, we observed that participants went through three successive stages when accomplishing strategic negotiation: Timing, Epistemic Action and Proposition Evaluation. 

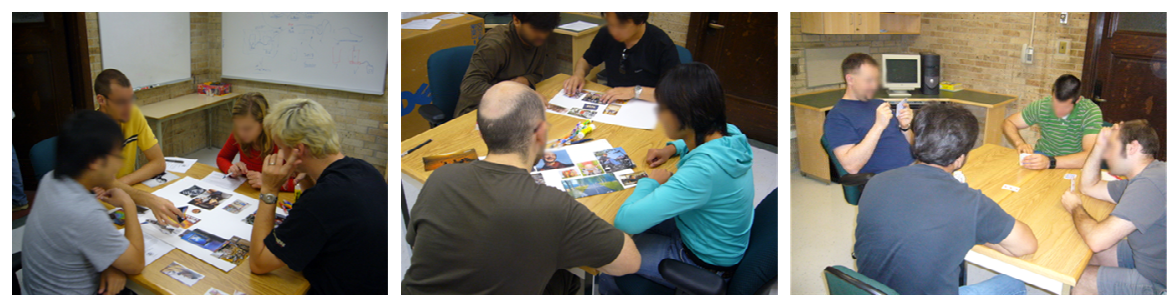

Fig. 1. Building a story in Task 1 (left), building stories in groups in Task 2 (middle), and playing a card game called "Pit" in Task 3 (right)

Timing: Capturing and retaining attention is important for strategic negotiations. In real-world setups, this is left to the charisma of the user. So users need to interrupt and gain others' attention to be able to efficiently negotiate. In Tasks 1 and 2, participants had to convince others to use as many of their own five selected images as possible. The center of the table (public space) and the spaces surrounding and between participants (shared space) were used to perform the main activities. During negotiation (when a participant suggested the use of his/her own image for the story), it was observed that the participant switched from his personal space to public space many times. For example, when the participants discussed transportation in their story, one participant suggested using an image of ships instead of an image of airplanes. He tried to gain the others' attention and negotiated strategically by moving the images back-and-forth between the personal and public spaces.

In Task 3, it was difficult for participants to be time efficient, because of the turntaking nature of the game. Participants could speculate on which suits were being collected by observing the card in the public space or the cards requested for exchange. Despite the limited value of timing in this task, we found that participants were conscious of the value of timing. Most often participants indicated their sense of urgency or preparedness by moving their next card (face-down) into a make-shift personal space.

Epistemic Actions: Kirsh and Maglio [5] introduced the idea of epistemic actions to understand how users perform certain actions to improve their cognition of the world. They argue that epistemic actions are physical actions that users perform to reduce the memory, number of steps or probability of error involved in a mental computation. In our task, we found that participants performed three types of epistemic actions: i) checking the details of images or the card's suit repeatedly, ii) shuffling the image with no apparent reason or rearranging card positions in order of suit in preparation for the next negotiation, and iii) hesitating and shuffling the image and card repeatedly just before starting a negotiation. All participants performed these actions, which enabled them to create a strategy or a plan-of-attack in several places.

We found that participants frequently performed epistemic actions before initiating or terminating a negotiation. For example, in Tasks 1 and 2, participants often arranged the images with the storyline in their mind and tried to advocate use of their own image through repeated shuffling motions during discussion of the story. In Task 3, we observed that participants checked a card's suit endlessly while awaiting their turn and also rearranged the card's position when comparing their own cards and the card 
indicated for exchange. They also rearranged the card's position after exchange in preparation for the next negotiation. They were often observed to hesitate or reconsider their move when starting the negotiation.

Proposition Evaluation: We found that there were two steps in evaluating the value of a proposition to the current negotiation. The initial step is a coarse grain evaluation of the value of the negotiation. It means that a decision is reached without much consideration, and the object of negotiation is either deemed to be potentially valuable or not interesting at the current moment. The second step is accepting that the object could have potential value and performing a detailed examination of its value to the current negotiation.

For example, in building a story, when a participant suggested a different plot for the story by using his own images, he tried to indicate his image to the others via hand and body gestures that pointed to the images in his own personal space. Other participants recognized his actions and evaluated his proposal in a two-step process. If the initial examination suggests that the proposal is attractive, he is allowed the opportunity to place the images in the public space (Task 1) or the shared space (Task 2), and then present his case to further the discussion and evaluation. These actions were often observed and considered to be a more useful method for efficient collaboration. In Task 3, the cards introduced for transaction were carefully placed in front of the other participant by the giving participant. The exchanged card was usually quickly evaluated in the receiving participant's personal space (fast initial evaluation). If this card was rejected, it was moved back to the personal space of the giving participant. However, if the card was not immediately rejected, it was moved to the private space where it was evaluated again, this time more carefully in comparison with the other cards in the hand. Then if this card was not accepted for exchange, it was moved back to the personal space of the giving participant.

Summary: In all tasks, we observed the above sequence of actions for strategic negotiations. In general, users rely a lot on their personal space to effectively time the negotiation and use various forms of hand and body gestures to draw attention to their proposition or evaluate another user's proposition.

\section{Study of Strategic Negotiations at Digital Table}

In order to further explore the sequence of actions for strategic negotiations and examine similarities and differences between digital and real-world tabletop negotiations, we conducted a similar study using digital setups.

\subsection{Tasks and Method}

Our study in digital setups involved observing six groups (four participants each) performing in the same setting. We carried out our observations using three digital tabletops inspired by existing systems. All experimental setups used a table of size $1261 \times 1530 \mathrm{sq} \mathrm{mm}$ and a horizontal display of size $635 \times 1030 \mathrm{sq} \mathrm{mm}$. Users could stand comfortably in front of the table. To keep the total experiment time to a reasonable amount, we restricted the task to Task 1 of the real-world setup. Similar to 
Task 1, 40 images were used for each task. Participants were only allowed to change image locations; no other image manipulations were allowed.

At the beginning of each session, the groups were given instructions indicating how to perform the task. Following the instructions, the participants had a threeminute practice session before beginning the experimental task on each setup. For each setup, the task duration was 15 minutes.

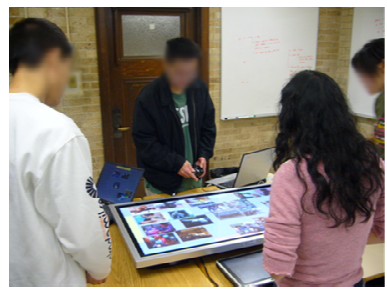

(a) UbiTable-Inspired

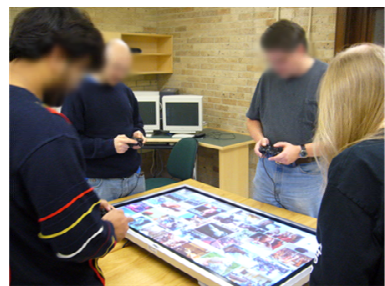

(b) DiamondTouch-Inspired

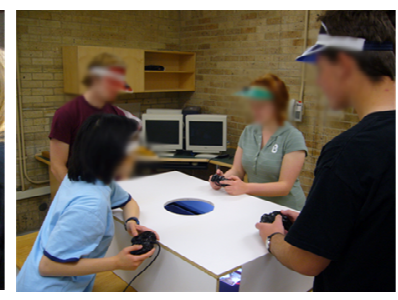

(c) SharedWell

Fig. 2. Three digital setups

\subsection{Tabletop Setups}

Figure 2 shows the different experimental setups on the table. The three digital tabletop systems were inspired by UbiTable [16], DiamondTouch [4] and the strategic negotiation table described in [6], which we call "SharedWell." These systems were chosen for their different use of private, personal and shared spaces.

UbiTable-Inspired: Figure 2(a) shows the digital setup inspired by the UbiTable system. This system uses four small computers (two notebooks and two tablet computers) as the users' private space, and each user's screen top is connected to a large display. Users can handle their contents on their own screen as private space and transfer them to the large display as public space by moving them to the top of their own screen. Essentially, users can use the large display and their own small computers seamlessly, but cannot access each other's small computers. This system did not support personal or shared spaces.

DiamondTouch-Inspired: This digital setup is inspired by the DiamondTouch system in the sense that it's a large public space without any private, personal or shared spaces. Figure 2(b) shows the setup using a large single display. Participants can see all of the contents and can control their own cursors via an input device.

SharedWell: Figure 2(c) shows the users, whose head positions are tracked, looking through an aperture in the table to view their digital contents. This aperture allows users to maintain a private view of their contents even when they move around the table. To show or share their own contents, users have to come close enough to each other so that their views through the aperture overlap. The overlapping region creates a shared space for users to show or share contents. If a user wants to show or share contents with a particular partner, he puts the contents in the overlapping area by approaching the partner.

In all systems, participants were divided according to four colors (red, green, blue and yellow) and could recognize their own images and cursor by the color. A game controller was used as an input device for each participant to control the digital 
contents. All sessions were videotaped and analyzed to compare and contrast the sharing of both the tabletop workspace and the objects on the table.

\subsection{Results}

Based on the analysis of the video and the image movements, we describe our observations on strategic negotiation in the different digital tables.

UbiTable-Inspired: Participants had 10 different images to view on their private screens (private spaces) at the beginning, where they often rearranged the images. These epistemic actions helped them create a storyline. Soon after determining the images to be used, some participants moved them to the public space as the selected images. However, we also observed that others kept relocating the images (including images to be used in their own storyline) in their own private spaces. As their recommendations, these participants gradually moved the images into the public space as the session progressed. We believe that they preserved these images in their own private spaces so as to strategize and wait for the right timing to present them. In addition, we also observed that when participants initiated a discussion or negotiation with the others, they pointed at their image in the public space by using their own cursor or hand. If the image was not accepted after the group evaluation (this is considered the second step of the evaluation), the owner typically moved it back to his/her private space.

DiamondTouch-Inspired: This setup allowed all participants to see and handle all images during the entire session. At the beginning, these 40 images were distributed to each participant and placed in front of her at the edge of the table. In order to create their own storylines, participants rearranged their images within the area where the images were delivered. After determining images to be used for their own storylines in their minds, they moved the unnecessary images to the corner of the table, whereas they left the necessary images stationary in front of them as the selected images. We noticed that all participants handled their images only in the public space. They did not wait for the appropriate timing to present a particular image to the others efficiently because they could see all the images all the time during the session. Here a participant had to move the images by a cursor, and this seemed to present cognitive uncertainty to the other participants about the operator's intentions and so on. The epistemic actions occurred in the form of redundant movements of images, however they were not effectively used among the participants because of the cognitive uncertainty of the cursor operation. From the observation, they seemed to be used only for the careful consideration of the participant. When a participant tried to propose a particular image to be used in the storyline shared by all participants, she moved the image from the area in front of her to the public space. If there were images forming a storyline in the public space, we often observed that a participant put his image on top of these images, occluding them. By using these actions he could initiate negotiation and force a group evaluation of the proposal. Evaluations were often done in a single step.

SharedWell: The SharedWell system was designed to improve strategic negotiation by supporting shared spaces. Because of the nature of this system, participants performed many more physical movements for initiating or starting negotiations than 
with any of the other systems investigated. At the beginning, we observed that participants positioned themselves away from the table, therefore, distances among participants were long enough to preserve their own private spaces, avoiding the overlapping of their spaces to create the shared spaces. When a participant tried to initiate negotiation or discussion, he moved himself closer to the other participant(s) to create a shared space. This space was used as the public space for the discussion and negotiation between the two (or sometimes more) participants. Actually, they initiated negotiation or discussion by moving images from their private spaces to the shared space when the timing was right. All of the actions mentioned here could be observed in all of the participants, and they demonstrated the epistemic actions as well. Soon after the images were moved to the shared space, others evaluated them quickly. This was the first step of evaluation and was repeated several times by changing partners. Through a sequence of negotiations, images were gradually collected in the public space to form a storyline. These images were finally reevaluated carefully. This was the second step of the evaluation process.

\section{Discussions}

From the results of the experiment, it is clear that there are differences in how participants engage in strategic negotiations in real-world and digital setups.

\subsection{Real-World Setups vs. Digital Tabletop Setups}

Based on the observations of the real-world setups and digital setups, we found many problems with the current digital tabletops. These problems relate to differences in user actions at different stages of strategic negotiation.

Timing: For strategic negotiation, users typically use their private spaces to examine information that needs to be shown to the other participants at the most appropriate time to maximize its impact. In the UbiTable-inspired system, we found that the participants could not use their private spaces effectively. We observed that some participants moved their selected images from the private space to the public space directly soon after the session started. Here, they did not create their own personal space explicitly, and thus they missed a chance to strategize and present information at the most appropriate time. In the DiamondTouch-inspired system, participants could not determine the best timing to propose their images since all information was visible to all participants from the beginning of the session. On the other hand, in the SharedWell system, participants could determine the most appropriate timing by observing the other participants' explicit movements; however, the physical movements forced participants to miss some opportune moments and at the same time quickly fatigued them.

Epistemic Actions: In the real-world settings, all participants easily noticed all negotiations and actions, including epistemic actions performed by a participant. Through these actions, a participant could understand the status of the collaboration taking place on the table, e.g. who was negotiating with whom, and results of the negotiation. At first we thought that the DiamondTouch-inspired system was the most similar to the real-world settings except that it required indirect manipulation using a 
cursor. However, we noticed that participants had difficulties in recognizing epistemic actions made by others because of the cognitive uncertainty of the cursor operation. On the other hand, in the other two systems, participants could perform the epistemic actions. In the UbiTable-inspired system, participants had their own private spaces and showed epistemic actions by rearranging their images while they considered their own storylines. Similarly, in the SharedWell system, they also had their own private spaces, and through physical motions such as enlarging the private space to be shared with adjacent participants or moving images from their private spaces to the shared spaces, they showed epistemic actions.

Proposition Evaluation: We found that users often evaluated a proposition by transitioning attention between spaces, especially from the private space to the personal space and from the personal space to the shared/public space. Explicit transitions between spaces attract other persons' attention and help provide cues of the evaluation process to all participants. From observations in the real-world settings, we understand that the two-step evaluation is important for the strategic negotiations. Moreover, through the investigation of digital tables, we found that the facility of providing personal spaces is especially important for proposition evaluation. The SharedWell system was designed to transfer the information between spaces because of the nature of this system. Therefore, participants could often perform the two-step evaluation efficiently. In the DiamondTouch-inspired system, participants could see and manipulate all the images on the display; therefore, they had to transfer the images by using explicit actions such as hand gestures or utterances. On the other hand, the participants on the UbiTable-inspired system moved their images from the private space to the public space without paying special attention to the timing; therefore, they often missed the chance to do a first-evaluation at the most appropriate time.

\subsection{Supporting Strategic Negotiations on Digital Table}

Digital tables can be made more efficient for strategic negotiation by improving various aspects of timing, epistemic actions and proposition evaluation.

Value of Personal Space: One of the crucial elements of strategic negotiation in real-world collaboration was a users' ability to maintain a personal space. Users often moved valuable negotiation data to the personal space, which served two purposes; first it informed others that this user had something that could be perceived as useful to the negotiation without giving them insights to evaluate the value of the data. This gave the user the opportunity to initiate negotiation when the moment was ripe. The second benefit of having information in the personal space was that at the right moment the user could easily introduce data to the public space for negotiation, and because others were anticipating this, they were more willing to listen to the user's proposition and were not taken by surprise. We believe that digital tables should support both personal and private spaces for enhanced strategic negotiations. Scott et al. $[13,14]$ suggest that digital tables do not have to support personal spaces because users generate these spaces by themselves. However, as described in the case of the UbiTable-inspired and DiamondTouch-inspired systems, very few participants created personal spaces because they either failed to appreciate their value or had insufficient workspace area. Systems could provide a default personal space that can 
be fluidly and intuitively moved around. We agree with Scott et al. [15] that it is important that users are able to flexibly and dynamically increase, decrease or relocate personal space within the workspace.

Sensitivity to User's Hand and Body Gestures: In the real-world task, users often created opportunities for negotiation by using various hand and body gestures. This rich communication language provided all users with awareness of each other's intentions allowing them to anticipate forthcoming actions. Hand and body gestures like rearranging cards within in the hand and drifting an image in-and-out of the public spaces provide rich awareness cues that users often pay attention to subconsciously. When a person tries to negotiate with others profitably, it is reasonable to expect this person to be aware of the other users' actions without compromising their privacy. It is this awareness of details that enables users to efficiently strategize negotiations in a group. Therefore, in order to support these negotiations, digital tables should be sensitive to users' actions related to body or hand gestures, and at the same time have the ability to keep private information private.

Interruptability and Epistemic Actions: We often observed that users interrupt each other with finesse to grab attention and propose an item for negotiation. While we did not explicitly examine interruption in our study we feel that digital systems should be proactive in providing support for interruptability. Systems could leverage a variety of multimodal information channels to further enhance strategic negotiation. As outlined in the results section, users rely on various epistemic actions to propose and evaluate negotiations. These could range from explicit transitions between spaces to attract other persons' attention to pondering and fiddling with the hands to indicate serious contemplation of the value of a proposition.

\subsection{Implications for Design}

The results of our investigation into strategic negotiations in digital tables have several implications for the design of future digital tabletop systems.

Support Creation of Personal Spaces: In our study of real-world strategic negotiations, we found that users often create personal spaces to negotiate efficiently. Tabletops that support strategic negotiations should not rely solely on private or public spaces and transfer information from private to public space directly. When designing strategic negotiations efficiently, an important implication is that the system must support creation of personal spaces.

Support Flexible and Fluid transition between Spaces: In all our digital systems, users repeatedly transferred information between private, personal and shared/public spaces. Fluid transition of information between these spaces is important for conducting strategic negotiations efficiently. Many researchers are exploring novel interaction techniques to support flexible and fluid transitions between different spaces. Our results reaffirm the need to do so.

Tabletop Systems should be Sensitive to Body and Hand Gestures: We observed that users relied on their hand and body gestures to negotiate in real-world setups. For strategic negotiation, these gestures also helped users to know the intentions of another other person's actions exactly. Therefore, we believe digital tables should be 
sensitive to these gestures while at the same time not demanding from users explicit gestures as with the SharedWell system. We believe that future systems must harness the body and hand gestures of users with greater finesse for strategic negotiations.

Provide Greater Support for Epistemic Actions: We repeatedly observed that users performed many epistemic actions during collaborations. While the digital tabletop systems did not explicitly factor in epistemic actions in their designs, the users were able to perform some of the epistemic actions observed in real-world settings. However, for digital tabletops to attain the flexibility and fluidity of real-world collaboration, we need to explicitly take into consideration typical epistemic actions when designing future tabletop systems.

\section{Conclusions and Future Work}

We investigated strategic negotiations in real-world face-to-face collaborations and compared the findings with three digital tabletop systems. We found that users strategize at multiple levels, preferring to use a personal space of dynamically rechanging size. We also identified several characteristics of group dynamics that can be valuable for designing next generation tabletop systems. Our results show that in the real-world, strategic negotiation involves three phases: identifying the right timing, using epistemic actions to draw attention and evaluating the value of the negotiation. We repeated the real-world experiments with different digital tabletops and found several differences in the way users initiate and perform strategic negotiations. In the future we plan to look into studying strategic negotiations with a variety of personalities and leadership qualities to see if there are any differences. We are also exploring novel ways to extend tabletop systems like the SharedWell to capture the nuances of negotiation that are evident in the real world.

\section{References}

1. Bui, T.X., Shakun, M.F.: Introduction of the negotiation support system minitrack. In: Proceedings of HISS, Hawaii, 23 (2000)

2. Elwart-Keys, M., Halonen, D., Horton, M., Kass, R., Scott, P.: User interface requirement for face to face groupware. In: Proceedings of the ACM CHI, pp. 295-301. ACM Press, New York (1990)

3. Dietmeyer, B., Kaplan, R.: Strategic negotiation: A breakthrough four-step process for effective business negotiation. Kaplan Business (2004)

4. Dietz, P., Leigh, D.: DiamondTouch: A multi-user touch technology. In: Proceedings of the 14th Annual ACM UIST, pp. 219-226. ACM Press, New York (2001)

5. Kirsh, D., Maglio, P.: On distinguishing epistemic from pragmatic action. Cognitive Science 18(4), 513-549 (1994)

6. Kitamura, Y., Osawa, W., Yamaguchi, T., Takemura, H., Kishino, F.: A display table for strategic collaboration preserving private and public information. In: Proceedings of IFIP ICEC 2005, pp. 167-179 (2005)

7. Kruger, R., Carpendale, S., Scott, S.D., Greenberg, S.: Roles of orientation in tabletop collaboration: comprehension, coordination and communication. Journal of Computer Supported Cooperative Work 13(5-6), 501-537 (2004) 
8. Matsushita, M., Iida, M., Ohguro, T., Shirai, Y., Kakehi, Y., Naemura, T.: Lumisight table: A face-to-face collaboration support system that optimizes direction of projected information to each stakeholder. In: Proceedings of ACM CSCW, pp. 274-283. ACM Press, New York (2004)

9. Patten, J., Ishii, H., Hines, J., Pangaro, G.: Sensetable: A wireless object tracking platform for tangible user interfaces. In: Proceedings of ACM CHI, pp. 253-260. ACM Press, New York (2001)

10. Pinelle, D., Gutwin, C., Greenberg, S.: Task analysis for groupware usability evaluation: modeling shared-workspace tasks with the mechanics of collaboration. ACM Transactions on Computer-Human Interaction 10(4), 281-311 (2003)

11. Rekimoto, J., Saitoh, M.: Augmented Surfaces: A spatially continuous work space for hybrid computing environments. In: Proceedings of ACM CHI, pp. 378-385. ACM Press, New York (2000)

12. Ryall, K., Forlines, C., Shen, C., Morris, M.R.: Exploring the effects of group size and table size on interactions with tabletop shared-display groupware. In: Proceedings of ACM CSCW, pp. 284-293. ACM Press, New York (2004)

13. Scott, S.D., Carpendale, S.T., Inkpen, K.M.: Territoriality in collaborative tabletop workspaces. In: Proceedings of ACM CSCW, pp. 294-303. ACM Press, New York (2004)

14. Scott, S.D., Carpendale, S.T., Habelski, S.: Storage Bins: Mobile storage for collaborative tabletop displays. IEEE Computer Graphics and Applications 25(4), 58-65 (2005)

15. Scott, S.D., Grant, K.D., Mandryk, R.L.: System guidelines for co-located, collaborative work on a tabletop display. In: Proceedings of ECSCW, pp. 159-178 (2003)

16. Shen, C., Everitt, K., Ryall, K.: UbiTable: Impromptu face-to-face collaboration on horizontal interactive surfaces. In: Dey, A.K., Schmidt, A., McCarthy, J.F. (eds.) UbiComp 2003. LNCS, vol. 2864, pp. 281-288. Springer, Heidelberg (2003)

17. Streitz, N., Geibler, J., Holmer, T., Konomi, S., Muller-Tomfelde, C., Reischl, W., Rexroth, P., Seitz, P., Steinmetz, R.: i-LAND: An interactive landscape for creativity and innovation. In: Proceedings of the ACM CHI, pp. 120-127. ACM Press, New York (1999)

18. Tandler, P., Prante, T., Müller-Tomfelde, C., Streitz, N., Steinmetz, R.: Connectables: Dynamic coupling of displays for the flexible creation of shared workspaces. In: Proceedings of the 14th Annual ACM UIST, pp. 11-20. ACM Press, New York (2001)

19. Tang, A., Tory, M., Po, B., Neumann, P., Carpendale, S.T.: Collaborative coupling over tabletop displays. In: Proceedings of ACM CHI, pp. 1181-1190. ACM Press, New York (2006)

20. Tse, E., Histon, J., Scott, S.D., Greenberg, S.: Avoiding interference: How people use spatial separation and partitioning in SDG workspaces. In: Proceedings of ACM CSCW, pp. 252-261. ACM Press, New York (2004)

21. Tyler, T.R., Blader, S.L.: Cooperation in groups: Procedural justice, social identity and behavioral engagement. Psychology Press (2000)

22. Ullmer, B., Ishii, H.: The metaDESK: Models and prototypes for tangible user interfaces. In: Proceedings of the 10th Annual ACM UIST, pp. 223-232. ACM Press, New York (1997)

23. Wu, M., Balakrishnan, R.: Multi-finger and whole hand gestural interaction techniques for multi-user tabletop displays. In: Proceedings of the 16th Annual ACM UIST, pp. 193-202. ACM Press, New York (2003) 